\title{
The role of boundary data on the solvability of some equations involving non-autonomous nonlinear differential operators
}

\section{Cristina Marcelli*}

"Correspondence:
marcelli@dipmat.univpm.it
Dipartimento di Ingegneria
Industriale e Scienze Matematiche,
Università Politecnica delle Marche,
Via Brecce Bianche, Ancona, 60131,
Italy

\begin{abstract}
The paper deals with the existence and non-existence of solutions of the following strongly nonlinear non-autonomous boundary value problem:

(P) $\left\{\begin{array}{l}\left(a(t, x(t)) \Phi\left(x^{\prime}(t)\right)\right)^{\prime}=f\left(t, x(t), x^{\prime}(t)\right) \quad \text { a.e. } t \in \mathbb{R}, \\ x(-\infty)=v^{-}, \quad x(+\infty)=v^{+}\end{array}\right.$

with $v^{-}<v^{+}$, where $\Phi: \mathbb{R} \rightarrow \mathbb{R}$ is a general increasing homeomorphism, with $\Phi(0)=0, a$ is a positive, continuous function and $f$ is a Caratheódory nonlinear function.

The same problem was already studied in the case when $|f(t, x, y) / \Phi(y)| \rightarrow 0$ as $y \rightarrow 0$ in the recent paper (Marcelli in Electron. J. Differ. Equ. 2012: 171, 2012), where sharp sufficient conditions for the existence or non-existence of solutions were established. In particular, it was proved that neither the behavior of the functions $a(t, \cdot)$ and $f(t, \cdot, y)$ nor the boundary data $v^{-}, v^{+}$influence the solvability of problem (P).

We herein study the critical case when $|f(t, x, y)| \sim|\Phi(y)|$ as $|y| \rightarrow 0$, focusing on the role played by the dependence on $x$ of the functions $a$ and $f$ and by the boundary data $v^{-}, v^{+}$by means of an explicit link between them and the other parameters of the differential equation.
\end{abstract}

MSC: Primary 34B40; 34C37; secondary 34B15; 34L30

Keywords: boundary value problems; unbounded domains; heteroclinic solutions; nonlinear differential operators; $p$-Laplacian operator; $\Phi$-Laplacian operator

\section{Introduction}

A wide literature has been devoted to the study of boundary value problems for differential equations involving various types of nonlinear differential operators. The most known are differential equations of the type

$$
\left(\Phi\left(x^{\prime}\right)\right)^{\prime}=f\left(t, x, x^{\prime}\right)
$$

governed by nonlinear differential operators such as the classical $p$-Laplacian or its generalizations. Various types of differential operators, even singular or non-surjective, have been considered due to many applications in different fields. We now quote just some of

\section{Springer}

○2013 Marcelli; licensee Springer. This is an Open Access article distributed under the terms of the Creative Commons Attribution License (http://creativecommons.org/licenses/by/2.0), which permits unrestricted use, distribution, and reproduction in any medium, provided the original work is properly cited. 
the papers devoted to this study, such as for the scalar case Bereanu and Mawhin [1, 2], Cabada and Pouso [3, 4], Cabada and Cid [5], Cid and Torres [6], Calamai [7], GarciaHuidobro et al. [8], Dang and Oppenheimer [9], Ferracuti and Papalini [10], O'Regan [11], Papageorgiou and Papalini [12]. In [13] Manásevich and Mawhin treated systems of equations with periodic boundary conditions. Finally, in the framework of differential inclusions, we quote [14] and the papers by Kyritsi, Matzakos and Papageorgiou [15, 16] for systems of differential inclusions involving maximal monotone operators and with various boundary conditions.

Besides differential operators acting on the derivative $x^{\prime}$, in many models such as reaction-diffusion equations (see, e.g., [17]) or porous media equations, differential operators involving the state variable $x$ also occur, and the study of mixed differential equations like

$$
\left(a(x) \Phi\left(x^{\prime}\right)\right)^{\prime}=f\left(t, x, x^{\prime}\right)
$$

has assumed a certain interest.

In [18] a periodic problem on a compact interval for a vectorial inclusion with a differential operator of the type $\left(a(x)\left\|x^{\prime}\right\|^{p-2} x^{\prime}\right)^{\prime}$ is studied, where $a: \mathbb{R} \rightarrow \mathbb{R}$ is a positive, continuous function. Moreover, in [16] a Dirichlet problem driven by a more general differential operator, having the structure $\left(A\left(x, x^{\prime}\right)\right)^{\prime}$, is investigated.

More recently, boundary value problems on the whole real line of the type

$$
\left\{\begin{array}{l}
\left(a(x(t)) \Phi\left(x^{\prime}(t)\right)\right)^{\prime}=f\left(t, x(t), x^{\prime}(t)\right) \quad \text { for a.e. } t \in \mathbb{R}, \\
x(-\infty)=v^{-}, \quad x(+\infty)=v^{+}
\end{array}\right.
$$

have been studied in [19], where existence and non-existence of solutions was put in relation to the behavior of $\Phi$ and $f(t, x, \cdot)$ at 0 and $f(\cdot, x, y)$ at infinity, while the presence of the function $a$ does not influence the existence of solutions. Subsequently, in [20] a critical case was considered in which also the dependence on the state variable $x$ of the functions $a$ and $f$ and the value of the boundary data are relevant for the solvability of the boundary value problem.

Finally, in the recent paper [21], non-autonomous differential operators were also considered, introducing the dependence on $t$ to the function $a$, that is, the following problem was proposed:

$$
\left\{\begin{array}{l}
\left(a(t, x(t)) \Phi\left(x^{\prime}(t)\right)\right)^{\prime}=f\left(t, x(t), x^{\prime}(t)\right) \quad \text { for a.e. } t \in \mathbb{R} \\
x(-\infty)=v^{-}, \quad x(+\infty)=v^{+}
\end{array}\right.
$$

with $v^{-}<v^{+}$given constants, where $\Phi: \mathbb{R} \rightarrow \mathbb{R}$ is a general increasing homeomorphism, with $\Phi(0)=0$, and $a$ is a positive, continuous function, but with possibly null infimum. It was shown that also the dependence on $t$ of the function $a$ plays a central role for the existence and non-existence of solutions and some sufficient criteria for the existence and nonexistence of solutions were established. However, in [21] the case when $|f(t, x, y) / \Phi(y)| \rightarrow 0$ as $y \rightarrow 0$ was considered, and in this setting neither the behavior with respect to $x$, nor the boundary data influence the existence or non-existence of solutions. 
The aim of this paper is to complete this study, investigating the critical case $|f(t, x, y)| \sim$ $|\Phi(y)|$ as $y \rightarrow 0$ for problem (1.1) governed by non-autonomous differential operators.

We provide sharp sufficient conditions guaranteeing the solvability of problem (1.1) together with conditions implying the non-existence of solutions, closely related to the former ones, involving the asymptotic behaviors of $a(\cdot, x)$ and $f(\cdot, x, y)$ as $|t| \rightarrow+\infty$, the asymptotic behaviors of $\Phi$ and $f(t, x, \cdot)$ as $y \rightarrow 0$, and the maxima/minima of the functions $a(t, \cdot), f(t, \cdot, y)$ in the interval $\left[v^{-}, v^{+}\right]$defined by the boundary data.

We present general existence and non-existence results (see Theorems 2.1, 2.2 and 2.3) together with operative criteria (see Propositions 3.1-3.6) useful when the functions $a$ and $f$ appearing in the differential equation have a product structure. Some examples of application complete the paper.

All the present results extend the ones contained in [20] to the case of non-autonomous differential operators. However, according to our knowledge, the results here presented are new even for $\Phi(y) \equiv y$, that is, for differential equations of the type (see [22])

$$
\left(a(t, x(t)) x^{\prime}(t)\right)^{\prime}=f\left(t, x(t), x^{\prime}(t)\right) \quad \text { a.e. } t .
$$

\section{Existence and non-existence theorems}

Throughout the paper, $\Phi$ is a general increasing homeomorphism on $\mathbb{R}$ such that $\Phi(0)=0$, $a: \mathbb{R}^{2} \rightarrow \mathbb{R}$ is a positive continuous function and $f: \mathbb{R}^{3} \rightarrow \mathbb{R}$ is a Carathéodory function.

Dealing with the nonlinear differential equation

$$
\left(a(t, x(t)) \Phi\left(x^{\prime}(t)\right)\right)^{\prime}=f\left(t, x(t), x^{\prime}(t)\right) \text { a.e. } t
$$

we will adopt the following notations:

$$
\begin{aligned}
& m(t):=\min _{x \in\left[v^{-} v^{+}\right]} a(t, x) M(t):=\max _{x \in\left[v^{-} v^{+}\right]} a(t, x), \\
& m^{*}(t):=\min _{(s, x) \in[-t, t] \times\left[v^{-} v^{+}\right]} a(s, x) M^{*}(t):=\max _{(s, x) \in[-t, t] \times\left[v^{-} v^{+}\right]} a(s, x) .
\end{aligned}
$$

Of course, $M^{*}(t) \geq M(t) \geq m(t) \geq m^{*}(t)>0$ for every $t \in \mathbb{R}$, with inf $t \in \mathbb{R} m(t)$ possibly null.

As we have mentioned in Introduction, in the present paper, we treat problems for which, roughly speaking, $|f(t, x, \cdot)| \sim|\Phi(y)|$ as $y \rightarrow 0$. But also the rate of growth of $\Phi$ at $\infty$ has a great relevance, and we separately consider the case of superlinear growth from that of linear or sublinear growth.

We first state an existence result for differential operators growing at most linearly at infinity.

Theorem 2.1 Let $\Phi$ be such that

$$
\limsup _{|y| \rightarrow+\infty} \frac{|\Phi(y)|}{|y|}<+\infty .
$$

Assume that

$$
f\left(t, v^{-}, 0\right) \leq 0 \leq f\left(t, v^{+}, 0\right) \quad \text { for a.e. } t \in \mathbb{R}
$$


and suppose that there exist constants $L, H>0$, a continuous function $\theta: \mathbb{R}^{+} \rightarrow \mathbb{R}^{+}$and a function $\lambda \in L^{q}([-L, L])$, with $1 \leq q \leq \infty$, such that

$$
\begin{aligned}
& |f(t, x, y)| \leq \lambda(t) \theta(a(t, x)|\Phi(y)|) \quad \text { for a.e. }|t| \leq L, \text { every } x \in\left[v^{-}, v^{+}\right],|y| \geq H, \\
& \int^{+\infty} \frac{\tau^{1-\frac{1}{q}}}{\theta(\tau)} \mathrm{d} \tau=+\infty
\end{aligned}
$$

(with $\frac{1}{q}=0$ if $\left.q=+\infty\right)$.

Finally, suppose that for every $C>0$, there exist a function $\eta_{C} \in L^{1}(\mathbb{R})$ and a function $\Lambda_{C} \in L_{\mathrm{loc}}^{1}([0,+\infty))$, null in $[0, L]$ and positive in $[L,+\infty)$, such that

$$
\begin{aligned}
& N_{C}(t):=\Phi^{-1}\left(\frac{M^{*}(L) \Phi(C)}{m(t)} e^{-\left|\int_{0}^{t} \frac{\Lambda_{C}(|s|)}{M(s)} d s\right|}\right) \in L^{1}(\mathbb{R}), \\
& \left\{\begin{array}{l}
f(t, x, y) \leq-\Lambda_{C}(t) \Phi(|y|), \\
f(-t, x, y) \geq \Lambda_{C}(t) \Phi(|y|)
\end{array} \quad \text { for a.e. } t \geq L, \text { every } x \in\left[v^{-}, v^{+}\right],|y| \leq N_{C}(t),\right. \\
& |f(t, x, y)| \leq \eta_{C}(t) \quad \text { if } x \in\left[v^{-}, v^{+}\right],|y| \leq N_{C}(t), \text { for a.e. } t \in \mathbb{R} .
\end{aligned}
$$

Then there exists a function $x \in C^{1}(\mathbb{R})$ such that $t \mapsto a(t, x(t)) \Phi\left(x^{\prime}(t)\right)$ belongs to $W^{1,1}(\mathbb{R})$ and

$$
\left\{\begin{array}{l}
\left(a(t, x(t)) \Phi\left(x^{\prime}(t)\right)\right)^{\prime}=f\left(t, x(t), x^{\prime}(t)\right) \quad \text { for a.e. } t \in \mathbb{R} \\
v^{-} \leq x(t) \leq v^{+} \quad \text { for every } t \in \mathbb{R} \\
x(-\infty)=v^{-}, \quad x(+\infty)=v^{+} .
\end{array}\right.
$$

Proof The scheme of the proof is the same as in [21, Theorem 3.1]. We sketch now the main points and prove in detail the parts which differ from that proof. Notice that there are only two differences between the present statement and that of [21, Theorem 3.1]; that is, we here take $\gamma=1$ and modify the definition of the auxiliary function $N_{C}$.

Fix $n \in \mathbb{N}, n>L$, and put $I_{n}:=[-n, n]$. Consider the truncation operator $T: W^{1,1}\left(I_{n}\right) \rightarrow$ $W^{1,1}\left(I_{n}\right)$ defined by

$$
T(x):=T_{x}, \quad \text { where } T_{x}(t):=\max \left\{v^{-}, \min \left\{v^{+}, x(t)\right\}\right\}
$$

and for every $x \in W_{\text {loc }}^{1,1}(\mathbb{R})$, put

$$
Q_{x}(t):=\max \left\{-N_{C}(t), \min \left\{T_{x}^{\prime}(t), N_{C}(t)\right\}\right\} .
$$

Finally, for every $x \in \mathbb{R}$, put $w(x):=\max \left\{x-v^{+}, 0\right\}+\min \left\{x-v^{-}, 0\right\}$.

Let us consider the following auxiliary boundary value problem on the compact interval $I_{n}$ :

$$
\left\{\begin{array}{l}
\left(a\left(t, T_{x}(t)\right) \Phi\left(x^{\prime}(t)\right)\right)^{\prime}=f\left(t, T_{x}(t), Q_{x}(t)\right)+\arctan (w(x(t))) \quad \text { a.e. in } I_{n}, \\
x(-n)=v^{-}, \quad x(n)=v^{+} .
\end{array}\right.
$$

Following the same argument in the proof of [21, Theorem 3.1], it is possible to prove that problem (2.11) admits a solution $u_{n}$ for every $n>L$, such that $v^{-} \leq u_{n}(t) \leq v^{+}$for all 
$t \in I_{n}$. Moreover, $u_{n}$ is increasing in $[-n,-L]$ and in $[L, n]$ and if $u_{n}^{\prime}\left(t_{0}\right)=0$ for some $\left|t_{0}\right|>L$, then $u_{n}^{\prime}(t)=0$ whenever $|t|>\left|t_{0}\right|$ (see Steps 1-2 in the proof of [21, Theorem 3.1]). Finally, as in Step 3 of the same proof, one can show that there exists a suitable constant $C$ such that $\left|u_{n}^{\prime}(t)\right|<C \leq N_{C}(t)$ for every $t \in[-L, L]$. Notice that till this point in the proof of [21, Theorem 3.1], the definition of $N_{C}$, or the fact that $\gamma>1$, were not used.

Now our goal is to show that $\left|u_{n}^{\prime}(t)\right| \leq N_{C}(t)$ also for every $t \in I_{n} \backslash[-L, L]$.

Since $u_{n}^{\prime}(t) \geq 0$ for every $t \in I_{n} \backslash[-L, L]$, to prove the claim it remains to show that $u_{n}^{\prime}(t) \leq$ $N_{C}(t)$ for every $t \in I_{n} \backslash[-L, L]$. To this aim, let $\hat{t}:=\sup \left\{t>L: u_{n}^{\prime}(\tau)<N_{C}(\tau)\right.$ in $\left.[L, t]\right\}$ and assume, by contradiction, $\hat{t}<n$. By the definition of $T$ and $Q_{u_{n}}$, we have

$$
\left(a\left(t, u_{n}(t)\right) \Phi\left(u_{n}^{\prime}(t)\right)\right)^{\prime}=f\left(t, T_{u_{n}}(t), Q_{u_{n}}(t)\right)=f\left(t, u_{n}(t), u_{n}^{\prime}(t)\right) \quad \text { a.e. in }[L, \hat{t}] .
$$

Since $u_{n}^{\prime}(t) \geq 0$ in $[L, n)$, by (2.9) we have

$$
\left(a\left(t, u_{n}(t)\right) \Phi\left(u_{n}^{\prime}(t)\right)\right)^{\prime} \leq-\Lambda_{C}(t) \Phi\left(u_{n}^{\prime}(t)\right) \leq-\frac{\Lambda_{C}(t)}{M(t)}\left(a\left(t, u_{n}(t)\right) \Phi\left(u_{n}^{\prime}(t)\right)\right)
$$

for a.e. $t \in[L, \hat{t}]$. Then, integrating in $[L, t)$ for $t \in[L, \hat{t})$, we obtain

$$
\begin{aligned}
a\left(t, u_{n}(t)\right) \Phi\left(u_{n}^{\prime}(t)\right) & \leq a\left(L, u_{n}(L)\right) \Phi\left(u_{n}^{\prime}(L)\right) e^{-\int_{L}^{t} \frac{\Lambda C_{C}(s)}{M(s)} \mathrm{d} s} \\
& <M^{*}(L) \Phi(C) e^{-\int_{L}^{t} \frac{\Lambda_{C}(s)}{M(s)} \mathrm{d} s}
\end{aligned}
$$

implying that $u_{n}^{\prime}(t)<N_{C}(t)$ for every $t \in[L, \hat{t}]$ (see (2.2), (2.3) and (2.8)), a contradiction when $\hat{t}<n$. So, $\hat{t}=n$ and the claim is proved. The same argument works in the interval $[-n,-L]$ too.

Therefore, we have $\left|u_{n}^{\prime}(t)\right| \leq N_{C}(t)$ for every $t \in[-n, n]$ implying that

$$
a\left(t, u_{n}(t)\right) \Phi\left(u_{n}^{\prime}(t)\right)=f\left(t, u_{n}(t), u_{n}^{\prime}(t)\right) \quad \text { a.e. in } I_{n} .
$$

Now, following the same argument as in [21, Theorem 3.1], it is possible to prove that the sequence $\left(\tilde{u}_{n}\right)_{n}$ of the functions $u_{n}$, continued in a constant way in the whole $\mathbb{R}$, converges to a solution $x$ of problem (1.1), satisfying all the properties stated in the assertion.

Similarly to what was done in [21], one can prove a result for differential operators having superlinear growth at infinity, provided that condition (2.7) is strengthened requiring that the Nagumo function has sublinear growth at infinity, as the following result states, whose proof is just the same as that of [21, Theorem 3.2], taking account of the modifications due to the different auxiliary function $N_{C}$, we showed in the proof of Theorem 2.1.

Theorem 2.2 Suppose that all the assumptions of Theorem 2.1 are satisfied, with the exception of (2.4), and with (2.7) replaced by

$$
\lim _{y \rightarrow+\infty} \frac{\theta(y)}{y}=0 .
$$

Then the assertion of Theorem 2.1 follows. 
Of course, the operators here considered as having superlinear growth are quite general and extend the classical $p$-Laplacian. Nevertheless, when dealing just with the $p$-Laplacian, the results can be slightly improved by using the positive homogeneity of the operator, as we will show in a forthcoming paper.

The key tools in the previous existence theorems is the summability of function $N_{C}(t)$ (condition (2.8)) joined with assumption (2.9). Such conditions are not improvable in the sense that if (2.9) is satisfied with the reversed inequality and $N_{C}$ is not summable, then problem (1.1) does not admit solutions, as the following result states.

Theorem 2.3 Suppose that there exist a constant $\rho>0$ and a positive function $\Lambda \in$ $L_{\mathrm{loc}}^{1}([0,+\infty))$ such that the following pair of conditions holds:

$$
\begin{aligned}
& f(t, x, y) \geq-\Lambda(t) \Phi(y) \quad \text { for a.e. } t \geq 0, \text { every } x \in\left[v^{-}, v^{+}\right], y \in(0, \rho), \\
& f(t, x, y) \leq \Lambda(-t) \Phi(y) \quad \text { for a.e. } t \leq 0, \text { every } x \in\left[v^{-}, v^{+}\right], y \in(0, \rho)
\end{aligned}
$$

and for every constant $C$, the function

$$
N_{C}(t):=\Phi^{-1}\left(\frac{C}{M(t)} e^{-\left|\int_{0}^{t} \frac{\Lambda(s \mid)}{m(s)} \mathrm{d} s\right|}\right)
$$

does not belong to $L^{1}(\mathbb{R})$.

Moreover, assume that

$$
t f(t, x, y) \leq 0 \quad \text { for a.e. } t \in \mathbb{R} \text {, every }(x, y) \in\left[v^{-}, v^{+}\right] \times \mathbb{R}
$$

and there exist two constants $\epsilon, H>0$ such that

$$
\begin{aligned}
& a\left(t, x_{1}\right) \leq H a\left(t+\delta, x_{2}\right) \quad \text { for every } t>0, x_{1}, x_{2} \in\left[v^{-}, v^{+}\right] \text {and } 0<\delta<\epsilon, \\
& a\left(t+\delta, x_{1}\right) \leq H a\left(t, x_{2}\right) \quad \text { for every } t<0, x_{1}, x_{2} \in\left[v^{-}, v^{+}\right] \text {and } 0<\delta<\epsilon .
\end{aligned}
$$

Then problem (1.1) does not admit solutions such that $v^{-} \leq x(t) \leq v^{+}$, that is, no function $x \in C^{1}(\mathbb{R})$, with $t \mapsto a(t, x(t)) \Phi\left(x^{\prime}(t)\right)$ almost everywhere differentiable, exists satisfying the conditions of problem (1.1).

Proof Also this proof follows the scheme of that of [21, Theorem 3.3]. More in detail, it is possible to show that if $x \in C^{1}(\mathbb{R})$ with $v^{-} \leq x(t) \leq v^{+}$and $a(t, x(t)) \Phi\left(x^{\prime}(t)\right)$ almost everywhere differentiable (not necessarily belonging to $W^{1,1}(\mathbb{R})$ ) is a solution of problem (1.1), then the function $x$ is monotone increasing in $[L,+\infty)$ and in $(-\infty,-L]$ with $\lim _{t \rightarrow \pm \infty} x^{\prime}(t)=0$.

Let us now define $t^{*}:=\inf \left\{t \geq 0: x^{\prime}(t)<\rho\right.$ in $\left.[t,+\infty)\right\}$ and assume, by contradiction, that $x^{\prime}\left(t^{*}\right)>0$. Put $T:=\sup \left\{t: x(t)<v^{+}\right\}$, so that $0<x^{\prime}(t)<\rho$ in $\left(t^{*}, T\right)$. By (2.13), for every $t \in\left(t^{*}, T\right)$, we get

$$
a(t, x(t)) \Phi\left(x^{\prime}(t)\right) \geq\left(a\left(t^{*}, x\left(t^{*}\right)\right) \Phi\left(x^{\prime}\left(t^{*}\right)\right)\right) e^{-\int_{t^{*}}^{t} \frac{\Lambda(s)}{m(s)} \mathrm{d} s}
$$


implying

$$
x^{\prime}(t) \geq \Phi^{-1}\left(\frac{a\left(t^{*}, x\left(t^{*}\right)\right) \Phi\left(x^{\prime}\left(t^{*}\right)\right)}{M(t)} e^{-\int_{t^{*}}^{t} \frac{\Lambda(s)}{m(s)} \mathrm{d} s}\right) .
$$

Then if $T<+\infty$, necessarily we have $x^{\prime}(T)=0$ in contradiction with the above inequality. Therefore, $T=+\infty$ and again, by the above inequality, we deduce $x(+\infty)=+\infty$ since by (2.15) the function on the right-hand side in not summable by assumption. Therefore, $x^{\prime}\left(t^{*}\right)=0$, implying that $t^{*}=0, x^{\prime}(t)=0$ for every $t \geq 0$ and, consequently, $x(0)=v^{+}$.

Similarly, using (2.14) one can show that $x(0)=v^{-}$, a contradiction.

\section{Some asymptotic criteria}

We devote this section to state some operative criteria which can usefully applied to operators and right-hand sides having the product structure

$$
a(t, x)=\alpha(t) \beta(x) \quad \text { and } \quad f(t, x, y)=b(t, x) c(x, y) .
$$

We will highlight how the local behaviors of $c(x, \cdot)$ at $y=0$ and of $b(\cdot, x), \alpha(\cdot)$ at infinity, related to the maximum and the minimum of the functions $\beta, g$ in the interval $\left[v^{-}, v^{+}\right]$, play a relevant role for the existence or non-existence of solutions.

In what follows, we assume that $\alpha, \beta$ are continuous positive functions, $b$ is a Carathéodory function and $c$ is a continuous function such that

$$
c(x, y)>0 \quad \text { for every } y \neq 0 \text { and } x \in\left[v^{-}, v^{+}\right] ; \quad c\left(v^{-}, 0\right)=c\left(v^{+}, 0\right)=0 .
$$

In this framework, putting $\tilde{m}:=\min _{x \in\left[v^{-}, v^{+}\right]} \beta(x)$ and $\tilde{M}:=\max _{x \in\left[v^{-}, v^{+}\right]} \beta(x)$, we have

$$
m(t)=\tilde{m} \alpha(t) \quad \text { and } \quad M(t)=\tilde{M} \alpha(t) \quad \text { for every } t \in \mathbb{R},
$$

where recall that $m(t):=\min _{x \in\left[v^{-}, v^{+}\right]} a(t, x)$ and $M(t):=\max _{x \in\left[v^{-}, v^{+}\right]} a(t, x)$.

Finally, we put

$$
m_{\infty}:=\inf _{t \in \mathbb{R}} \alpha(t) \geq 0 .
$$

\subsection{Case of $\Phi$ growing at most linearly}

In this subsection, we deal with differential operators $\Phi$ satisfying condition (2.4) that is such that $|\Phi(y)| \leq \Lambda|y|$ whenever every $|y|>H$ for some $H, \Lambda>0$. With this class of operators, we cover differential equations of the type

$$
\left(a(t, x(t)) x^{\prime}(t)\right)^{\prime}=f\left(t, x(t), x^{\prime}(t)\right) .
$$

The first two existence theorems are an application of Theorem 2.1.

\section{Proposition 3.1 Suppose that}

$$
t \cdot b(t, x)<0 \quad \text { for a.e. } t \text { such that }|t| \geq L \text {, every } x \in\left[v^{-}, v^{+}\right]
$$


for some $L \geq 0$, and there exists a function $\lambda \in L_{\mathrm{loc}}^{q}(\mathbb{R}), 1 \leq q \leq+\infty$, such that

$$
|b(t, x)| \leq \lambda(t) \quad \text { for a.e. } t \in \mathbb{R}, \text { every } x \in\left[v^{-}, v^{+}\right]
$$

Moreover, assume that there exists a real constant $p$ (not necessarily positive) such that for every $x \in\left[v^{-}, v^{+}\right]$, we have

$$
\begin{aligned}
& h_{1}|t|^{p} \leq \alpha(t) \leq h_{2}|t|^{p} \quad \text { a.e. }|t|>L, \\
& \lambda_{1}|t|^{p-1} \leq|b(t, x)| \leq \lambda_{2}|t|^{p-\sigma} \quad \text { a.e. }|t|>L, \\
& c(x, y) \geq k_{1} \Phi(|y|) \quad \text { for every } y \in \mathbb{R}, \\
& c(x, y) \leq k_{2} \Phi(|y|)^{\delta} \quad \text { whenever }|y|<\rho, \\
& c(x, y) \leq k_{2}|\Phi(y)|^{2-\frac{1}{q}} \quad \text { whenever }|y|>H
\end{aligned}
$$

for certain positive constants $h_{1}, h_{2}, \lambda_{1}, \lambda_{2}, k_{1}, k_{2}, \sigma, \delta, \rho, H$ such that $\delta \leq 1$,

$$
\begin{aligned}
& 1-\frac{\lambda_{1} k_{1}}{h_{2} \tilde{M}} \delta+p(1-\delta)<\sigma \leq 1, \\
& \lambda_{1} k_{1}+p h_{2} \tilde{M}>0
\end{aligned}
$$

where recall that $\tilde{M}=\max \beta(x)$ for $x \in\left[v^{-}, v^{+}\right]$. Finally, let condition (2.4) be satisfied and assume that

$$
\limsup _{y \rightarrow 0^{+}} \frac{\Phi(y)}{y^{\mu}}>0
$$

for some positive constant $\mu$ satisfying

$$
\mu<\frac{\lambda_{1} k_{1}}{h_{2} \tilde{M}}+p
$$

Then problem (1.1) admits solutions.

Proof Put $\theta(r):=k_{2}\left(\frac{r}{m^{*}(L)}\right)^{2-\frac{1}{q}}$ for $r>0$. From (3.3) and (3.8), it is immediate to verify the validity of conditions (2.6) and (2.7).

Put

$$
\Lambda(t):=k_{1} \min \left\{\min _{x \in\left[v^{-}, v^{+}\right]} b(-t, x),-\max _{x \in\left[v^{-}, v^{+}\right]} b(t, x)\right\} \quad \text { for } t>L
$$

and $\Lambda(t):=0$ for $0 \leq t \leq L$. By condition (3.3), we have $\Lambda \in L_{\text {loc }}^{1}([0,+\infty))$ and by (3.2) we have $\Lambda(t)>0$ for $t>L$. Observe that by (3.6) it follows that

$$
f(t, x, y)=b(t, x) c(x, y) \leq k_{1} b(t, x) \Phi(|y|) \leq-\Lambda(t) \Phi(|y|)
$$

and

$$
f(-t, x, y)=b(-t, x) c(x, y) \geq k_{1} b(-t, x) \Phi(|y|) \geq \Lambda(t) \Phi(|y|)
$$


for a.e. $t \geq L$, every $x \in\left[v^{-}, v^{+}\right]$and every $y \in \mathbb{R}$. Then condition (2.9) of Theorem 2.1 holds.

Now, from (3.5) it follows that $\lambda_{1} k_{1}|t|^{p-1} \leq \Lambda(|t|)$ for a.e. $|t| \geq L$ and by (3.4), recalling that $M(t)=\tilde{M} \alpha(t)$, we deduce that

$$
\frac{\Lambda(|t|)}{M(t)} \geq \frac{\lambda_{1} k_{1}}{h_{2} \tilde{M}} \frac{1}{|t|} \quad \text { whenever }|t|>L .
$$

So,

$$
\left|\int_{0}^{t} \frac{\Lambda(|\tau|)}{M(\tau)} \mathrm{d} \tau\right| \geq \frac{\lambda_{1} k_{1}}{h_{2} \tilde{M}} \log \frac{|t|}{L} \quad \text { whenever }|t|>L .
$$

Hence, for every fixed $C>0$, the function $N_{C}(t)$ defined in (2.8) satisfies

$$
\Phi\left(N_{C}(t)\right) \leq \text { Const. }|t|^{-\left(\frac{\lambda_{1} k_{1}}{h_{2}}+p\right)} \text { for }|t| \text { large enough. }
$$

By (3.10) we get $N_{C}(t) \rightarrow 0$ as $|t| \rightarrow+\infty$, and therefore by (3.11) we deduce

$$
N_{C}(t) \leq \text { Const. }|t|^{-\frac{1}{\mu}\left(\frac{\lambda_{1} k_{1}}{h_{2} \bar{M}}+p\right)} \text { for }|t| \text { large enough, }
$$

implying that $N_{C}(t) \in L^{1}(\mathbb{R})$ by (3.12). Then (2.8) holds too.

Since $\lim _{|t| \rightarrow+\infty} N_{C}(t)=0$, a constant $L_{C}^{*}>L$ exists such that $N_{C}(t) \leq \rho$ for every $|t| \geq L_{C}^{*}$. Let us define $\hat{C}:=\max _{|t| \leq L_{C}^{*}} N_{C}(t)$ and

$$
\eta_{C}(t):= \begin{cases}\max _{x \in\left[\nu^{-}, v^{+}\right]}|b(t, x)| \cdot \max _{(x, y) \in\left[v^{-}, v^{+}\right] \times[-\hat{C}, \hat{C}]} c(x, y) & \text { if }|t| \leq L_{C}^{*}, \\ \lambda_{2} k_{2}|t|^{p-\sigma} \Phi\left(N_{C}(t)\right)^{\delta} & \text { if }|t|>L_{C}^{*} .\end{cases}
$$

By (3.5) and (3.7), for a.e. $t \in \mathbb{R}$, for every $x \in\left[v^{-}, v^{+}\right]$and every $y \in \mathbb{R}$ such that $|y| \leq N_{C}(t)$, we have

$$
|f(t, x, y)|=|b(t, x)| c(x, y) \leq \eta_{C}(t)
$$

that is, condition (2.10). It remains to prove that $\eta_{C} \in L^{1}(\mathbb{R})$.

By (3.3) and the continuity of the function $c$, we have $\eta_{C} \in L^{1}\left(\left[-L_{C}^{*}, L_{C}^{*}\right]\right)$. Moreover, when $|t|>L_{C}^{*}$, by (3.13), we have

$$
\eta_{c}(t) \leq \text { Const. }|t|^{-\sigma-\frac{\lambda_{1} k_{1}}{h_{2} \bar{M}} \delta+p(1-\delta)}
$$

implying that $\eta_{c}(t) \in L^{1}\left(\mathbb{R} \backslash\left[-L_{C}^{*}, L_{C}^{*}\right]\right)$ by assumption (3.9).

Therefore, Theorem 2.1 applies and guarantees the assertion of the present result.

Remark 3.2 The introduction of the constants $\sigma$ and $\delta$ serves to state the result in the most general form, but often they can be taken both equal to 1 , in such a way that assumption (3.9) is trivially verified.

If $m_{\infty}>0$ (see (3.1)), condition (3.6) can be weakened, requiring that it holds only for $|y|$ small enough, as the following result states. 
Proposition 3.3 Let all the assumptions of Proposition 3.1 be satisfied, with the exception of (3.6) replaced by

$$
c(x, y) \geq k_{1} \Phi(|y|) \quad \text { whenever }|y|<\rho .
$$

Moreover, assume that $m_{\infty}>0$. Then problem (1.1) admits solutions.

Proof For every $C>0$, put

$$
\begin{aligned}
& \Gamma_{C}:=\max \left\{\rho, \Phi^{-1}\left(\frac{M^{*}(L)}{m_{\infty}} \Phi(C)\right)\right\}, \quad \hat{m}_{C}:=\min _{(x, y) \in\left[\nu^{-}, \nu^{+}\right] \times\left[\rho, \Gamma_{C}\right]} c(x, y), \\
& h_{C}:=\min \left\{k_{1}, \frac{\hat{m}_{C}}{\Phi\left(\Gamma_{C}\right)}\right\}
\end{aligned}
$$

and finally put

$$
\Lambda_{C}(t):=h_{C} \min \left\{\min _{x \in\left[\nu^{-}, \nu^{+}\right]} b(-t, x),-\max _{x \in\left[\nu^{-}, \nu^{+}\right]} b(t, x)\right\}
$$

for $t>L$ and $\Lambda_{C}(t):=0$ for $t \in[0, L]$.

As it is immediate to verify, $c(x, y) \geq h_{C} \Phi(|y|)$ whenever $v^{-} \leq x \leq v^{+}$and $|y| \leq \Gamma_{C}$. So, (2.9) holds since $N_{C}(t) \leq \Gamma_{C}$ for every $t>L$.

From now on, the proof proceeds as that of Proposition 3.1.

We state now two non-existence results, obtained applying Theorem 2.3.

\section{Proposition 3.4 Suppose that}

$$
t \cdot b(t, x) \leq 0 \quad \text { for a.e. } t \in \mathbb{R} \text { and every } x \in\left[v^{-}, v^{+}\right],
$$

and let there exist a constant $p \in \mathbb{R}, a$ constant $L>0$ and a positive function $\ell(t) \in L^{1}([0, L])$ such that

$$
\begin{aligned}
& |b(t, x)| \leq \lambda_{2}|t|^{p-1} \quad \text { for every } x \in\left[v^{-}, v^{+}\right] \text {a.e. }|t|>L, \\
& |b(t, x)| \leq \ell(|t|) \quad \text { for a.e. }|t| \leq L, x \in\left[v^{-}, v^{+}\right], \\
& c(x, y) \leq k_{2} \Phi(y) \quad \text { for every } x \in\left[v^{-}, v^{+}\right], 0<y<\rho
\end{aligned}
$$

for some positive constants $\lambda_{2}, k_{2}, \rho$. Moreover, assume that (3.4) holds for some constants $h_{1}, h_{2}, p$ such that

$$
\lambda_{2} k_{2}+h_{1} p \tilde{m}>0
$$

where recall that $\tilde{m}=\min \beta(x)$ for $x \in\left[v^{-}, v^{+}\right]$.

Furthermore, suppose that

$$
\limsup _{y \rightarrow 0} \frac{\Phi(y)}{y^{\mu}}<+\infty
$$


for some positive constant $\mu$ satisfying

$$
\mu \geq \frac{\lambda_{2} k_{2}}{h_{1} \tilde{m}}+p
$$

Finally, suppose that there exist two constants $\epsilon, H>0$ such that

$$
\begin{aligned}
& \alpha(t) \leq H \alpha(t+r) \quad \text { for every } t>0 \text { and } 0<r<\epsilon, \\
& \alpha(t+r) \leq H \alpha(t) \quad \text { for every } t<0 \text { and } 0<r<\epsilon .
\end{aligned}
$$

Then problem (1.1) does not admit solutions.

Proof First of all, notice that assumption (3.15) implies condition (2.16) and assumptions (3.22) and (3.23) respectively imply conditions (2.17), (2.18).

Putting

$$
\Lambda(t):= \begin{cases}k_{2} \ell(t) & \text { for } t \in[0, L] \\ \lambda_{2} k_{2} t^{p-1} & \text { for } t>L,\end{cases}
$$

we have that $\Lambda$ is a positive function belonging to $L_{\text {loc }}^{1}([0,+\infty))$ and one can easily verify that conditions (3.16), (3.17) and (3.18) guarantee the validity of (2.13) and (2.14). Moreover, by (3.4) we get

$$
\frac{\Lambda(|t|)}{\alpha(t)} \leq \frac{\lambda_{2} k_{2}}{h_{1}} \frac{1}{|t|} \quad \text { for }|t| \text { large enough. }
$$

Hence, if $N_{C}(t)$ is the function defined in (2.15), we have

$$
\Phi\left(N_{C}(t)\right) \geq \text { Const. }|t|^{-\frac{\lambda_{2} k_{2}}{h_{1} \tilde{m}}-p} \text { for }|t| \text { large enough. }
$$

By assumption (3.19), we get $N_{C}(t) \rightarrow 0$ as $|t| \rightarrow+\infty$ and by virtue of (3.20), we obtain

$$
N_{C}(t) \geq \text { Const. }|t|^{-\frac{1}{\mu}\left(\frac{\lambda_{2} k_{2}}{h_{1} \tilde{m}}+p\right)}>0 \quad \text { for }|t| \text { large enough. }
$$

Finally, assumption (3.21) implies that $N_{C}(t)$ is not summable in $\mathbb{R}$ and the assertion follows as an application of Theorem 2.3.

Remark 3.5 As for the validity of conditions (3.22), (3.23) in the previous non-existence theorem, notice that when dealing with autonomous operators, that is, for $\alpha(t) \equiv 1$, they are trivially satisfied. However, also in the non-autonomous case, they hold in many relevant situations. For instance, they are satisfied if one the following conditions is satisfied:

$\alpha(t)$ is decreasing in $(-\infty, 0)$ and increasing in $(0,+\infty)$;

$\alpha$ is uniformly continuous in $\mathbb{R}$ and $\inf _{t \in \mathbb{R}} \alpha(t)>0$;

$\alpha(t) \sim|t|^{-p}$ as $|t| \rightarrow+\infty$ for some $p>0$.

When condition (3.19) does not hold, we can use the following non-existence result. 
Proposition 3.6 Let all the assumptions of Proposition 3.4 be satisfied with the exception of (3.20), (3.21) and with assumption (3.19) replaced by the opposite one,

$$
\lambda_{2} k_{2}+h_{1} p \tilde{m} \leq 0
$$

then problem (1.1) does not admit solutions.

Proof With the same notations of the proof of Proposition 3.4, notice that under condition (3.25), by (3.24), we have $N_{C}(t) \geq$ Const. $>0$ for $|t|$ large enough, implying that $N_{C}$ is not summable and the assertion follows from Theorem 2.3.

Let us now provide some examples of applications of the previous results.

Example 3.7 Let us consider the differential equation

$$
\left(|t|^{p} \beta(x) x^{\prime}(t)\right)^{\prime}=-\frac{t}{1+t^{2}}|t|^{p} g(x)\left|x^{\prime}(t)\right|,
$$

with $\beta, g$ positive continuous functions.

It is easy to show that all the assumptions of Proposition 3.1 are satisfied with $p>-2$, $q=1, h_{1}=h_{2}=k_{1}=k_{2}=1, \lambda_{1}<\min _{x \in\left[v^{-}, \nu^{+}\right]} g(x):=m_{g}, \lambda_{2}=\max _{x \in\left[v^{-}, v^{+}\right]} g(x):=M_{g}, p>-\frac{m_{g}}{\tilde{M}}$, $\sigma=\delta=\mu=1$, and $L$ large enough (depending on $m_{g}-\lambda_{1}$ ).

By applying Propositions 3.1 and 3.4, we deduce that if $p>1-\frac{m_{g}}{\tilde{M}}$, then problem (1.1) admits solutions, whereas if $p \leq 1-\frac{M_{g}}{\tilde{m}}$, then (1.1) does not admit solutions. Recalling that $\tilde{M}=\max \beta(x)(\tilde{m}=\min \beta(x))$ for $x \in\left[v^{-}, v^{+}\right]$, the existence or non-existence of solutions depends on the boundary data $v^{-}, v^{+}$. For instance, if $\beta(x):=\left(1+x^{2}\right)$ and $g(x):=e^{-|x|}$ and the boundary data are symmetric, that is, $v^{+}=-v^{-}=v$, then $M_{g}=\tilde{m}=1, \tilde{M}=1+v^{2}$ and $m_{g}=e^{-\nu}$. So, if $p>1-\frac{1}{e^{\nu}\left(1+\nu^{2}\right)}$, problem (1.1) admits solutions, whereas if $p \leq 0$, it does not admit solutions. Notice that for every $p>0$, problem (1.1) is solvable for $v$ small enough.

Example 3.8 Let us consider the differential equation

$$
\left(\frac{\beta(x)}{1+t^{2}} x^{\prime}(t) \arctan x^{\prime}(t)\right)^{\prime}=-\frac{t}{1+t^{4}} g(x) x^{\prime}(t)^{2},
$$

with $\beta, g$ positive continuous functions.

As one can immediately verify, assumptions (3.2)-(3.9) and (3.11) of Proposition 3.1 hold with $q=+\infty, p=-2, h_{1}<1, h_{2}=1, \lambda_{1}<\min _{x \in\left[v^{-}, v^{+}\right]} g(x):=m_{g}, \lambda_{2}=\max _{x \in\left[v^{-}, v^{+}\right]} g(x):=M_{g}$, $k_{1}=1, k_{2}>1, \sigma=\delta=1, \mu=2$ and $L, H$ large enough. Therefore, if $m_{g}>4 \tilde{M}$, both conditions (3.10) and (3.12) are satisfied and problem (1.1) admits solutions. Instead, if $M_{g} \leq 4 \tilde{m}$, then (1.1) has no solutions as a consequence of Proposition 3.4.

So, as in the previous example, the above conditions for the existence and non-existence of solutions become conditions on the boundary data $v^{-}, v^{+}$.

\subsection{Case of $\Phi$ having superlinear growth}

We handle now operators $\Phi$ having possibly superlinear growth at infinity, that is, we now remove condition (2.4). The non-existence Propositions 3.4 and 3.6 hold also in this case, since they do not require condition (2.4). As for the existence results, we now use 
Theorem 2.2 instead of Theorem 2.1 by assuming (2.12). As it will be clear after the proof of the next result, condition (2.12) is not satisfied when $m_{\infty}=0$, so from now on we assume $m_{\infty}>0$.

Proposition 3.9 Let all the assumptions of Proposition 3.3 hold true, with the exception of (3.8) replaced by

$$
\lim _{|y| \rightarrow+\infty} \frac{\max _{x \in\left[v^{-}, v^{+}\right]} c(x, y)}{|\Phi(y)|}=0 .
$$

Then problem (1.1) admits solutions.

Proof Put

$$
\theta(r):=\max _{(t, x) \in[-L, L] \times\left[v^{-}, v^{+}\right]}\left(\max \left\{c\left(x, \Phi^{-1}\left(\frac{r}{a(t, x)}\right)\right), c\left(x, \Phi^{-1}\left(-\frac{r}{a(t, x)}\right)\right)\right\}\right),
$$

it is immediate to check that $\theta$ is a continuous function on $[0,+\infty)$ such that

$$
\theta(a(t, x)|\Phi(y)|) \geq c(x, y) \quad \text { for every } t \in[-L, L], x \in\left[v^{-}, v^{+}\right], y \in \mathbb{R},
$$

hence (2.6) holds. Moreover, by (3.26), for every $\epsilon>0$, there exists a real $c_{\epsilon}$ such that

$$
c(x, y) \leq \epsilon|\Phi(y)| \quad \text { for every } x \in\left[v^{-}, v^{+}\right],|y| \geq c_{\epsilon} .
$$

Hence, for every $s \geq M^{*}(L) \max \left\{\Phi\left(c_{\epsilon}\right),-\Phi\left(-c_{\epsilon}\right)\right\}$, we have $\theta(s) \leq \frac{\epsilon}{m^{*}(L)} s$, that is,

$$
\lim _{s \rightarrow+\infty} \frac{\theta(s)}{s}=0
$$

Hence, condition (2.12) holds and the proof proceeds as that of Proposition 3.3, applying Theorem 2.2 instead of Theorem 2.1.

Note that condition (3.26) is not compatible with (3.6). For this reason, in the case of superlinear growth, we only treat the case $m_{\infty}>0$.

Example 3.10 Let us consider the following differential equation:

$$
\left((1+|t|) \beta(x) x^{\prime}(t) e^{\left|x^{\prime}(t)\right|}\right)^{\prime}=-\frac{t}{|t|} g(x)\left|x^{\prime}(t)\right|\left(1+x^{\prime}(t)^{2}\right)
$$

with $\beta, g$ positive continuous functions.

In this case, we can apply Proposition 3.9 since $m_{\infty}>0$ and condition (3.26) is trivially satisfied. Moreover, all the other assumptions of Proposition 3.9 hold with $q=+\infty$, $h_{1}=1, h_{2}>1, p=1, \lambda_{1}=\min _{x \in\left[v^{-}, v^{+}\right]} g(x), \lambda_{2}=\max _{x \in\left[v^{-}, v^{+}\right]} g(x), k_{1}<1<k_{2}, \mu=1$. Hence, since (3.12) is satisfied whatever $\lambda_{1}, \tilde{M}>0$ may be, problem (1.1) admits solutions for every boundary data $v^{-}, v^{+}$. 


\section{Competing interests}

The author declares that she has no competing interests.

Received: 13 May 2013 Accepted: 24 September 2013 Published: 22 Nov 2013

\section{References}

1. Bereanu, C, Mawhin, J: Boundary-value problems with non-surjective $\Phi$-Laplacian and one-side bounded nonlinearity. Adv. Differ. Equ. 11, 35-60 (2006)

2. Bereanu, C, Mawhin, J: Existence and multiplicity results for some nonlinear problems with singular $\Phi$-Laplacian. J. Differ. Equ. 243, 536-557 (2007)

3. Cabada, A, Pouso, RL: Existence results for the problem $\left(\phi\left(u^{\prime}\right)\right)^{\prime}=f\left(t, u, u^{\prime}\right)$ with periodic and Neumann boundary conditions. Nonlinear Anal. 30, 1733-1742 (1997)

4. Cabada, A, Pouso, RL: Existence results for the problem $\left(\phi\left(u^{\prime}\right)\right)^{\prime}=f\left(t, u, u^{\prime}\right)$ with nonlinear boundary conditions. Nonlinear Anal. 35, 221-231 (1999)

5. Cabada, A, Cid, JA: Heteroclinic solutions for non-autonomous boundary value problems with singular $\Phi$-Laplacian operators. Discrete Contin. Dyn. Syst. 2009, suppl., 118-122 (2009)

6. Cid, JA, Torres, PJ: Solvability for some boundary value problems with $\Phi$-Laplacian operators. Discrete Contin. Dyn. Syst. 23, 727-732 (2009)

7. Calamai, A: Heteroclinic solutions of boundary value problems on the real line involving singular $\Phi$-Laplacian operators. J. Math. Anal. Appl. 378(2), 667-679 (2011)

8. Garcia-Huidobro, M, Manásevich, R, Zanolin, F: Strongly nonlinear second-order ODE's with rapidly growing terms. J. Math. Anal. Appl. 202, 1-26 (1996)

9. Dang, H, Oppenheimer, SF: Existence and uniqueness results for some nonlinear boundary value problems. J. Math Anal. Appl. 198, 35-48 (1996)

10. Ferracuti, L, Papalini, F: Boundary value problems for strongly nonlinear multivalued equations involving different $\Phi$-Laplacians. Adv. Differ. Equ. 14, 541-566 (2009)

11. O'Regan, D: Existence theory for $\left(\phi\left(y^{\prime}\right)\right)^{\prime}=q f\left(t, y, y^{\prime}\right), 0<t<1$. Commun. Appl. Anal. 1, 33-52 (1997)

12. Papageorgiou, NS, Papalini, F: Pairs of positive solutions for the periodic scalar $p$-Laplacian. J. Fixed Point Theory Appl. 5, 157-184 (2009)

13. Manásevich, R, Mawhin, J: Periodic solutions for nonlinear systems with p-Laplacian-like operators. J. Differ. Equ. 145, 367-393 (1998)

14. Ferracuti, L, Marcelli, C, Papalini, F: Boundary value problems for highly nonlinear inclusions governed by non-surjective $\Phi$-Laplacians. Set-Valued Var. Anal. 19(1), 1-21 (2011)

15. Kyritsi, S, Matzakos, N, Papageorgiou, NS: Periodic problems for strongly nonlinear second-order differential inclusions. J. Differ. Equ. 183, 279-302 (2002)

16. Krytsi, ST, Matzakos, N, Papageorgiou, NS: Nonlinear boundary value problems for second order differential equations. Czechoslov. Math. J. 55, 545-579 (2005)

17. Maini, PK, Malaguti, L, Marcelli, C, Matucci, S: Diffusion-aggregation processes with mono-stable reaction terms. Discrete Contin. Dyn. Syst., Ser. B 6(5), 1175-1189 (2006)

18. Papageorgiou, EH, Papageorgiou, NS: Strongly nonlinear, multivalued, periodic problems with maximal monotone terms. Differ. Integral Equ. 17, 443-480 (2004)

19. Cupini, G, Marcelli, C, Papalini, F: Heteroclinic solutions of boundary value problems on the real line involving general nonlinear differential operators. Differ. Integral Equ. 24(7-8), 619-644 (2011)

20. Cupini, G, Marcelli, C, Papalini, F: On the solvability of a boundary value problem on the real line. Bound. Value Probl. 2011, 26 (2011)

21. Marcelli, C: Existence of solutions to some boundary-value problems governed by general nonlinear non-autonomous differential operators. Electron. J. Differ. Equ. 2012, 171 (2012)

22. Marcelli, C, Papalini, F: Heteroclinic connections for fully nonlinear non-autonomous second order differential equations. J. Differ. Equ. 241, 160-183 (2007)

10.1186/1687-2770-2013-252

Cite this article as: Marcelli: The role of boundary data on the solvability of some equations involving non-autonomous nonlinear differential operators. Boundary Value Problems 2013, 2013:252

\section{Submit your manuscript to a SpringerOpen ${ }^{\ominus}$ journal and benefit from:}

- Convenient online submission

Rigorous peer review

- Immediate publication on acceptance

- Open access: articles freely available online

- High visibility within the field

- Retaining the copyright to your article 